\title{
Klein tunneling in graphene p-n-p junctions
}

\author{
E. Rossi ${ }^{1}$, J. H. Bardarson ${ }^{2,3}$, P. W. Brouwer ${ }^{4}$ \\ ${ }^{1}$ Department of Physics, College of William and Mary, Williamsburg, VA 23187 , \\ USA \\ ${ }^{2}$ Department of Physics, University of California, Berkeley, California 94720, USA \\ ${ }^{3}$ Materials Sciences Division, Lawrence Berkeley National Laboratory, Berkeley, \\ California 94720, USA \\ ${ }^{4}$ Dahlem Center for Complex Quantum Systems and Institut für Theoretische \\ Physik, Freie Universität Berlin, Arnimallee 14, 14195 Berlin, Germany \\ We study the effect of the Klein tunneling on the transport prop- \\ erties of graphene p-n-p junctions. We first analyze the ideal case \\ of a clean system and then study the realistic case in which dis- \\ order is present. To take into account the effect of disorder we \\ develop a method for obtaining quantum transport properties in \\ graphene that uniquely combines three crucial features: micro- \\ scopic treatment of charge disorder, fully quantum mechanical \\ analysis of transport, and the ability to model experimentally \\ relevant system sizes. Our results allow us to conclude unam- \\ biguously that recent transport experiments on graphene p-n-p \\ junctions have indeed observed signatures of Klein tunneling. \\ The analysis presented is based on Ref. (1).
}

\section{Introduction}

In 1929 Oskar Klein discovered that relativistic particles can, at normal incidence, tunnel perfectly through a potential barrier with height comparable to their rest energy (2). This effect is now known as Klein tunneling (3). Up to this day the quest for an experimental verification of Klein tunneling of elementary particles has been unsuccessful. The recent experimental realization of graphene (4) has, however, opened up a completely new avenue for the exploration of Klein tunneling. This is due to the fact that the low energy electronic excitations of graphene are properly described as massless Dirac fermions. It has therefore been proposed that in a graphene p-n-p junction the transport should exhibit direct signatures of Klein tunneling (5). However, the interpretation of the experimental results is complicated by the unavoidable presence of disorder.

The doping level of graphene, $n_{\mathrm{bg}}$, can be controlled using a back-gate. When a top-gate is added a p-n-p junction can be defined electrostatically, Fig. 1. In the ideal case (absence of disorder) the inversion of polarity due to the top-gate creates an electrostatic barrier at the p-n and n-p interfaces. The electronic transmission through such a double barrier has a strong angular dependence due to Klein tunneling $(6,7)$ and for certain incident angles the electrons can be confined between the two barriers $(8,9)$. Resonant tunneling through these confined states leads to pronounced oscillations in the resistance as a function of system parameters, such as the top-gate voltage $(8,10,9)$. Early experiments failed to reproduce the predicted resistance 
oscillations $(11,12)$, presumably because of too much disorder (13). More recent experiments that seek to minimize the effect of disorder have been more successful (14, 15, 16). However, a theoretical understanding of whether, or to what extent, these experiments are really observing Klein tunneling phenomena, and the issue of the experimental conditions needed to see Klein tunneling unambiguously have remained open.

\section{Theoretical approach}

In this work the top gate is taken to have length $L_{\mathrm{tg}}=30 \mathrm{~nm}$ and to be placed at a distance $d_{\mathrm{tg}}=10 \mathrm{~nm}$ from the graphene layer. We assume the top gate to be in air with a dielectric constant $\epsilon_{1}=1$, and for the substrate we assume a dielectric constant $\epsilon_{2}=4$, corresponding to $\mathrm{SiO}_{2}$. These values reflect realistic experimental conditions. We perform our calculations for a square sample of length $L=160 \mathrm{~nm}$.

There is compelling evidence that in exfoliated graphene charge impurities are the main source of disorder $(17,18,19,20,21)$. Due to the vanishing density of states close to the Dirac point, even taking into account screening effects, the disorder potential created by the charge impurities retains its long-range nature $(22,23,24)$. This fact prevents the use of standard approaches to calculate the electronic structure and the transport properties of graphene in the presence of charge impurities. A very efficient method to obtain the ground state of graphene in the presence of Coulomb disorder is the Thomas-Fermi-Dirac theory (25). In this approach, similar in spirit to the Density Functional Theory, the energy functional, $E[n]$, specific for graphene in the presence of disorder, is minimized with respect to the density $n(\mathbf{r})$. We have:

$$
E[n]=\int d^{2} \mathbf{r} n(\mathbf{r})\left[\frac{2}{3} \hbar v_{F}|\pi n(\mathbf{r})|^{1 / 2}+V_{\mathrm{sc}}(\mathbf{r})\right]+E_{\mathrm{xc}}[n]
$$

where $v_{F} \approx 10^{6} \mathrm{~m} / \mathrm{s}$ is the Fermi velocity for graphene,

$$
V_{\mathrm{sc}}(\mathbf{r})=\hbar v_{F} r_{s}\left[V_{\mathrm{d}}(\mathbf{r})+V_{\mathrm{tg}}(\mathbf{r})+\frac{1}{2} \int d^{2} r^{\prime} \frac{n\left(\mathbf{r}^{\prime}\right)}{\left|\mathbf{r}-\mathbf{r}^{\prime}\right|}\right]-\hbar v_{F} \mu,
$$

with $r_{s} \equiv e^{2} / \hbar v_{F} \epsilon, V_{\mathrm{d}}$ and $V_{\mathrm{tg}}$ the potentials induced by the impurity density distribution, $C(\mathbf{r})$, and the top gate, respectively, and $\mu$ the chemical potential. $E_{\mathrm{xc}}[n]$ is the exchange correlation energy $(25,26)$. The charge impurities are assumed to be located in a $2 \mathrm{D}$ plane at a distance $d=1 \mathrm{~nm}$ from the graphene layer, (21), and to be in equal numbers positive and negative so that, denoting by angle brackets disorder averaged quantities, we have $\langle C(\mathbf{r})\rangle=0$. The impurities are also assumed to be uncorrelated so that $\left\langle C\left(\mathbf{r}_{1}\right) C\left(\mathbf{r}_{2}\right)\right\rangle=n_{\text {imp }} \delta\left(\mathbf{r}_{2}-\mathbf{r}_{1}\right)$, where $n_{\text {imp }}$ is the charge impurity density. The minimization is subject to the constraint $\left(1 / A^{\prime}\right) \int_{A^{\prime}} n(\mathbf{r}) d^{2} r=n_{\mathrm{bg}}$, where $A^{\prime}$ is the area of the sample where the top gate potential is negligible. The electrostatic potential $V_{\mathrm{tg}}$ is expressed in terms of the top gate charge density $n_{\mathrm{tg}}$

$$
V_{\mathrm{tg}}(\mathbf{r})=\int d^{2} \mathbf{r}^{\prime} \frac{n_{\mathrm{tg}}\left(\mathbf{r}^{\prime}\right)}{\left(\left|\mathbf{r}-\mathbf{r}^{\prime}\right|^{2}+d_{\mathrm{tg}}^{2}\right)^{1 / 2}},
$$

and is obtained self-consistently, for a fixed voltage difference between the top gate and graphene, $\varphi_{\mathrm{tg}}$, by requiring that in the region below the top gate $\Delta n_{\mathrm{tg}} \equiv n_{\mathrm{tg}}(\mathbf{r})-$ 

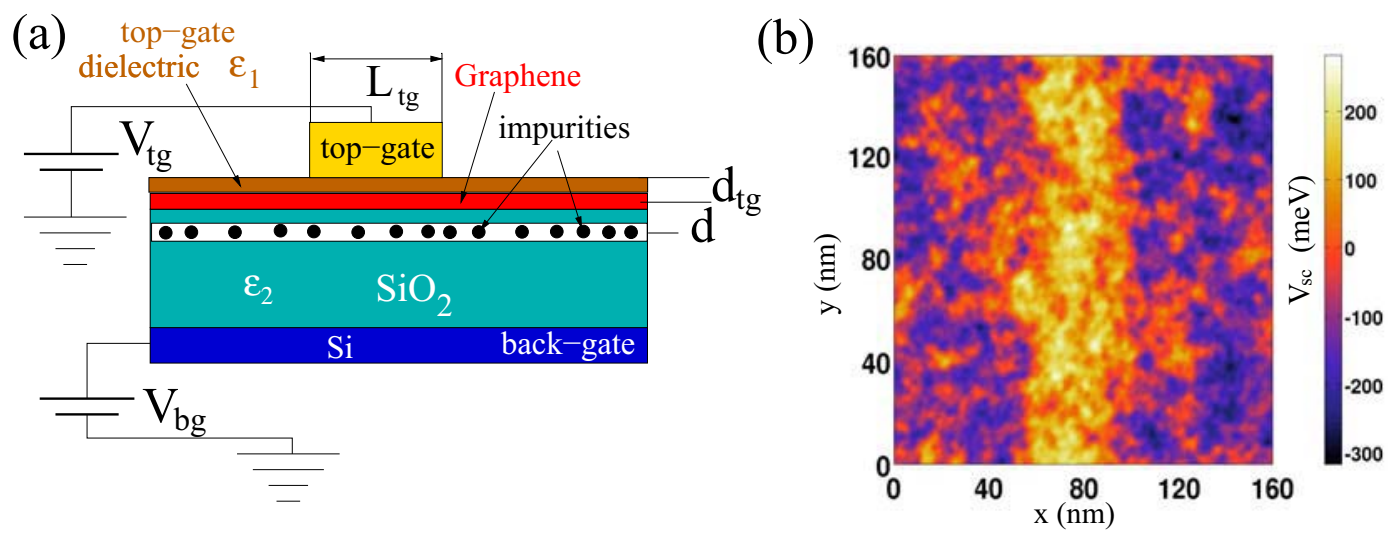

Figure 1: (a) A schematic of the top gated setup. Graphene is represented by the red layer and the top-gate dielectric by the brown one. (b) $V_{\mathrm{sc}}(\mathbf{r})$ for a disordered junction with $n_{\mathrm{bg}}=5 \times 10^{11} \mathrm{~cm}^{-2}, \Delta n_{\mathrm{tg}}=2 \times 10^{12} \mathrm{~cm}^{-2}$, and $n_{\mathrm{imp}}=5 \times 10^{11} \mathrm{~cm}^{-2}$. Adapted from Rossi et al. (1).

$n(\mathbf{r})=C_{\mathrm{tg}} \varphi_{\mathrm{tg}}$, where $C_{\mathrm{tg}}$ is the top gate capacitance. $\Delta n_{\mathrm{tg}}$ is the physical parameter that we vary to simulate a change of the potential applied to the top-gate.

By minimizing $E[n]$ the ground state carrier density $n(\mathbf{r})$ can be obtained as well as the screened potential $V_{\mathrm{sc}}(\mathbf{r})$. Fig. 1 (b) shows an example of $V_{\mathrm{sc}}(\mathbf{r})$ for a single disorder realization. In the presence of disorder the carrier density landscape breaks up into electron-hole puddles $(27,28,25,29,30)$ and the potential barriers between regions with opposite polarity are no longer well defined.

To calculate the electrical conductance we use a transfer-matrix approach $(31,32)$. In the presence of the disordered potential $V_{\mathrm{sc}}$ the Hamiltonian

$$
H=v_{F} \mathbf{p} \cdot \sigma+V_{\mathrm{sc}}(\mathbf{r})
$$

with $\sigma=\left(\sigma_{x}, \sigma_{y}\right)$ the Pauli matrices, defines a scattering problem. The Schrödinger equation $H \psi=0$ generates the transfer matrix $\mathcal{M}$, which relates the wavefunction at $x=0$ to the one at $x=L, \psi_{L}=\mathcal{M} \psi_{0} . \mathcal{M}$ is obtained numerically dividing the interval $(0, L)$ into $N$ equal subintervals of length $\delta x=L / N$ and calculating the transfer matrix in each subinterval via the Born approximation. This gives (32)

$$
\mathcal{M}=\prod_{n=1}^{N} e^{-\frac{i}{2} \delta x \partial_{y} \sigma_{z}} e^{-i u_{n} \sigma_{x}} e^{-\frac{i}{2} \delta x \partial_{y} \sigma_{z}} ; \quad u_{n}(y)=\frac{1}{\hbar v_{F}} \int_{(n-1) \delta x}^{n \delta x} d x V_{\mathrm{sc}}(x, y)
$$

In the limit $N \rightarrow \infty$ the Born approximation becomes exact. From the transfer matrix $\mathcal{M}$ we calculate the matrix $t$ of transmission amplitudes, which in turn gives us the two terminal conductance $G=R^{-1}=\left(4 e^{2} / h\right) \operatorname{tr} t t^{\dagger}$.

\section{Results}

We now present our results. Fig. 2 (a) is a color plot that shows how the resistance depends on $\Delta n_{\mathrm{tg}}$ and $n_{\mathrm{bg}}$. Quasi periodic oscillations as a function of $\Delta n_{\mathrm{tg}}$ are clearly visible. Fig. 2 (b) shows $R$ as function of $\Delta n_{\text {tg }}$ for fixed $n_{\mathrm{bg}}$ in the clean limit. The dashed line is the result for a single boundary condition along the transverse direction: 

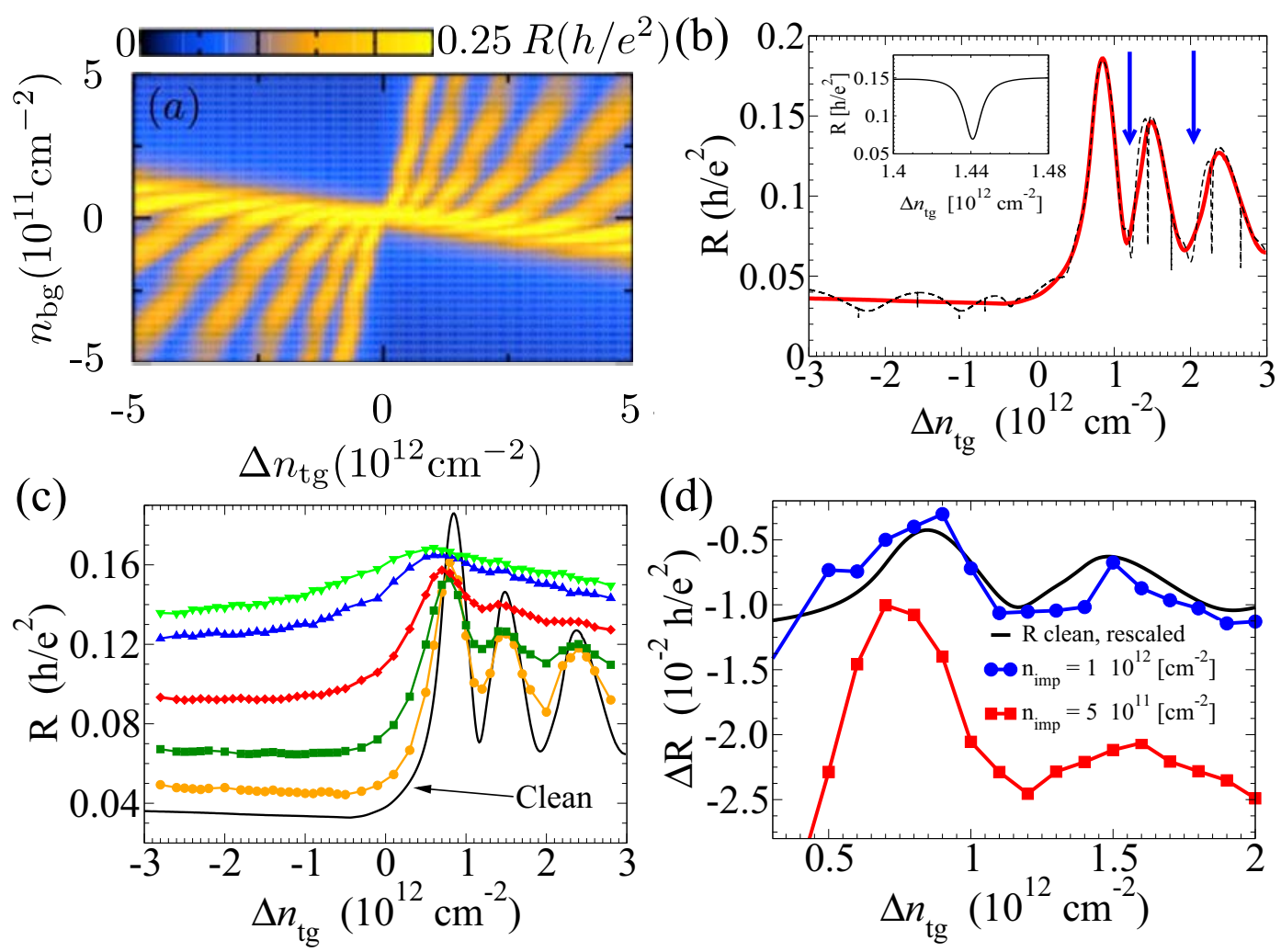

Figure 2: (a) Gate voltage dependence of the resistance for a clean device. (b) A single trace of the resistance at fixed back gate density $n_{\mathrm{bg}}=5 \times 10^{11} \mathrm{~cm}^{-2}$. The smooth curve is obtained by averaging over boundary conditions. The insets show a close up of one of the narrow resonances obtained for transverse periodic boundary condition. (c) Disorder averaged resistance as a function of $\Delta n_{\mathrm{tg}}$ for different values of $n_{\text {imp }}$ : from bottom to top $n_{\text {imp }}=0,1,2.5,5,10$, and $15 \times 10^{11} \mathrm{~cm}^{-2}$. (d) The resistance difference $\Delta R$ where we have subtracted the resistance at $n_{\text {imp }}=1.510^{12} \mathrm{~cm}^{-2}$ where the transport is diffusive, to emphasize the survival of broad oscillations for $n_{\text {imp }}$ as high as $10^{12} \mathrm{~cm}^{-2}$. Adapted from Rossi et al. (1).

in addition to broad oscillation sharp resonances can be observed due to resonant scattering via bound states in the region under the top-gate. The sharp resonances disappear when the results are averaged over different transverse boundary conditions (solid line).

Fig. 2 (c), (d) show our results in the presence of disorder. The results were obtained averaging over several - 1000 - disorder realizations. The narrow resonances disappear at very low impurity densities. On the other hand the broad oscillations, signature of Klein tunneling, survive for relatively high impurity densities. This can be observed clearly in Fig. 2 (d) from which we can see that, for our parameter values, the broad oscillations are observables for densities as high as $n_{\mathrm{imp}}=10^{12} \mathrm{~cm}^{-2}$.

The disordered averaged results can be understood by estimating the mean free path, $l$, in the presence of Coulomb disorder. When $l$ is of the order or smaller than the width, $W$, of the sample, scattering events start to dominate and the sharp resonances disappear. For the parameter values used we find that the sharp resonances disappear for $l \approx 400 \mathrm{~nm}$, i.e. of the order of $W=160 \mathrm{~nm}$. The broad oscillations are 
analogous to the oscillations of a Fabry-Perot interferometer in which the p-n and the $n-p$ junctions play the role of the reflecting surfaces. As a consequence, after disorder averaging, the broad oscillations survive as long as the mean free path is longer than the distance between the $\mathrm{p}-\mathrm{n}$ and the $\mathrm{n}-\mathrm{p}$ junction. Consistently we find that for our parameter values the broad oscillations can no longer be identified for impurity densities higher than the value corresponding to $l=40 \mathrm{~nm}$, the width of the $\mathrm{n}$ region created by the top-gate. This also explains the fact that the oscillations at high topgate voltages, for which the width of the n-region is wider, become unidentifiable at lower impurity densities than the oscillations at low $V_{\text {tg }}$. Very recent experiments (16) have found results in very good quantitative agreement with our predictions.

\section{Summary}

We have presented a powerful theoretical approach for calculating the transport properties of graphene in the presence of long-range disorder taking into account quantum interference effects. We have applied the method to study the transport properties of graphene p-n-p junctions. Our results identify the critical values of the disorder below which quantum effects are observable in transport measurements. Our results show unambiguously that recent transport experiments on graphene p-n-p junctions have observed clear signatures of the Klein tunneling, for the first time since its theoretically prediction, more than 80 years ago.

\section{References}

1. E. Rossi, J. H. Bardarson, P. W. Brouwer, and S. Das Sarma, Phys. Rev. B 81, 121408R (2010).

2. O. Klein, Z. Phys. 53, 157 (1929).

3. N. Dombey and A. Calogeracos, Phys. Rep. 315, 41 (1999).

4. K. S. Novoselov, A. K. Geim, S. V. Morozov, D. Jiang, Y. Zhang, S. V. Dubonos, I. V. Grigorieva, and A. A. Firsov, Science 306, 666 (2004).

5. M. I. Katsnelson, K. S. Novoselov, and A. K. Geim, Nat. Phys. 2, 620 (2006).

6. T. Ando, T. Nakanishi, and R. Saito, J. Phys. Soc. Japan 67, 2857 (1998).

7. V. Cheianov and V. Fal'ko, Phys. Rev. B 74, 041403 (2006).

8. P. G. Silvestrov and K. B. Efetov, Phys. Rev. Lett. 98, 016802 (2007).

9. J. H. Bardarson, M. Titov, and P. W. Brouwer, Phys. Rev. Lett. 102, 226803 (2009).

10. A. V. Shytov, M. S. Rudner, and L. S. Levitov, Phys. Rev. Lett. 101, 156804 (2008).

11. B. Huard, J. A. Sulpizio, N. Stander, K. Todd, B. Yang, and D. GoldhaberGordon, Phys. Rev. Lett. 98, 236803 (2007). 
12. N. Stander, B. Huard, and D. Goldhaber-Gordon, Phys. Rev. Lett. 102, 026807 (2009).

13. M. M. Fogler, D. S. Novikov, L. I. Glazman, and B. I. Shklovskii, Phys. Rev. B 77, 075420 (2008).

14. R. V. Gorbachev, A. S. Mayorov, A. K. Savchenko, D. W. Horsell, and F. Guinea, Nano Letters 8, 1995 (2008).

15. A. Young and P. Kim, Nature Physics 5, 222 (2009).

16. M. Begliarbekov, O. Sul, N. Ai, E.-H. Yang, and S. Strauf, App. Phys. Lett. 97, 122106 (2010).

17. Y.-W. Tan, Y. Zhang, K. Bolotin, Y. Zhao, S. Adam, E. H. Hwang, S. Das Sarma, H. L. Stormer, and P. Kim, Phys. Rev. Lett. 99, 246803 (2007).

18. S. Adam, E. H. Hwang, V. M. Galitski, and S. Das Sarma, Proc. Natl. Acad. Sci. USA 104, 18392 (2007).

19. J. H. Chen, C. Jang, S. Adam, M. S. Fuhrer, E. D. Williams, and M. Ishigami, Nature Physics 4, 377 (2008).

20. E. Rossi, S. Adam, and S. D. Sarma, Phys. Rev. B 79, 245423 (2009).

21. S. Das Sarma, S. Adam, E. H. Hwang, and E. Rossi, arXiv:1001.4731v2, to bepublished in Rev. Mod. Phys. (2010).

22. K. Nomura and A. H. MacDonald, Phys. Rev. Lett. 96, 256602 (2006).

23. T. Ando, J. Phys. Soc. Jpn. 75, 074716 (2006).

24. V. Cheianov and V. Fal'ko, Phys. Rev. Lett. 97, 226801 (2006).

25. E. Rossi and S. Das Sarma, Phys. Rev. Lett. 101, 166803 (2008).

26. M. Polini, A. Tomadin, R. Asgari, and A. MacDonald, Phys. Rev. B 78, 115426 (2008).

27. E. H. Hwang, S. Adam, and S. Das Sarma, Phys. Rev. Lett. 98, 186806 (2007).

28. J. Martin, N. Akerman, G. Ulbricht, T. Lohmann, J. H. Smet, K. von Klitzing, and A. Yacobi, Nature Physics 4, 144 (2008).

29. Y. Zhang, V. Brar, C. Girit, A. Zettl, and M. Crommie, Nature Physics 5, 722 (2009).

30. A. Deshpande, W. Bao, F. Miao, C. N. Lau, and B. J. LeRoy, Phys. Rev. B 79, 205411 (2009).

31. M. Titov, Europhys. Lett. 79, 17004 (2007).

32. J. H. Bardarson, J. Tworzydlo, P. W. Brouwer, and C. W. J. Beenakker, Phys. Rev. Lett. 99, 106801 (2007). 